\title{
Bindungsbiografisches Coaching zur achtsamen Gestaltung von Beziehungen im digitalen Kapitalismus
}

\author{
Michael Krohn ${ }^{1}$ \\ Online publiziert: 22. Juli 2020 \\ (c) Der/die Autor(en) 2020
}

\section{Zusammenfassung}

Dieser Beitrag der Zeitschrift Coaching | Theorie \& Praxis untersucht die Nutzbarmachung der modernen Bindungsforschung für das Coaching insbesondere in berufsbiografischen Übergängen und Arbeitssituationen, die von hoher Unsicherheit geprägt sind. Die interdisziplinäre Bindungsforschung kann hier wertvolle Impulse liefern, die bislang in Coachingforschung und -praxis kaum beachtet worden sind. Sie fragt nach den individuellen Faktoren, welche die psychische Sicherheit erhöhen und zwischenmenschliches Vertrauen entstehen lassen. Vertrauen gilt als wichtiges Element einer modernen Arbeitsorganisation. Dies weist auf eine Lücke bei der Erforschung des Zusammenhangs von Arbeit und Bindung hin. Trotz vieler offenen Fragen steht erstes Wissen für ein mentalisierungsgestütztes Coaching bereit. Es kann durch Achtsamkeit wirksam unterstützt werden. Hierzu werden erste Ansätze und Forschungsergebnisse vorgestellt und in einen eigenen Coachingansatz integriert. Im zunehmend ökonomisierten Gesundheitssystems kann anhand einer Methodendemonstration gezeigt werden, dass Ärzte/innen dadurch Trauer und Schuldgefühle in ihrer Berufsbiografie besser bewältigen können.

Schlüsselwörter New Work · Identitätsarbeit · Bindung · Achtsamkeit · Biographie · Mentalisierung

\section{Attachment based biographical coaching for the mindful arrangement of relationships in digital capitalism}

\begin{abstract}
This article in the journal Coaching I Theorie \& Praxis examines the utilization of modern attachement research for coaching particulary in regard to transitions within professional biography and work situations which are characterized by high uncertainty. Interdisciplinary attachment research can provide valuable impulses which have barely been acknowledged in coaching theory and practice. It poses the question to the individual factors which enhance psychological security and givet rise to interpersonal trust. Such trust is considered to be a central factor of modern work organisation. This indicates that theres is a gap in research of the connection between work and attachment. Despite many unanswered questions there is first knowledge for mentalization-based coaching. It can be effectively supported by mindfulness. For this purpose initial approaches and research findings are presented and integrated into an own coaching approach. Within an increasingly business-oriented healthcare system a demonstration of the method can show that this makes it easier for physicians to successfully cope with grief and feeling of guilt in their professional biography.
\end{abstract}

Keywords New work $\cdot$ Identity work $\cdot$ Attachment $\cdot$ Mindfulness $\cdot$ Biography $\cdot$ Mentalization

Michael Krohn

michael.krohn@med.uni-goettingen.de

1 Studiendekanat, Universitätsmedizin Göttingen, Robert-Koch-Straße 40, 37075 Göttingen, Deutschland
1 Herausforderungen der Entwicklungsaufgabe „Partnerwahl“ im digitalen Kapitalismus

Die Erforschung von Übergängen im Lebenslauf erhält in den Sozialwissenschaften derzeit eine größere Bedeutung, da sich in Folge seiner Entstandardisierung und zunehmen- 
der Biografisierung neue individuelle Unsicherheiten ergeben. Der analytische Fokus verschiebt sich von der traditionellen Frage des Verlaufes von festen normierten und linearen Übergängen und ihren Effekten auf deren biografisches Zustandekommen im Sinne der psychologisch betrachteten Entwicklung der Persönlichkeit im Lebenslauf. Dies wirft im Zusammenhang mit der Einbindung in soziale Netzwerke und Beziehungen auch die Frage auf, wie die selbstreflexive Gestaltung der erforderlichen Bildungsprozesse mit Hilfe von professioneller Beratung situationsspezifisch gelingen kann (Wanka et al. 2020). Aus einer Coachingperspektive werden die beruflichen Übergänge im Lebenslauf relevant, die sich aus den modernen Arbeitswelten ergeben.

Die Unternehmen stellen sich den modernen Herausforderungen einer immer komplexeren Umwelt durch verstärkte soziale Vernetzung im Rahmen einer „New Work“. In den modernen Organisationsentwicklungskonzepten ist der einst kapitalismuskritische und emanzipatorische Bildungscharakter dieses Konzeptes mittlerweile verloren gegangen, und „New Work“ dreht sich im Kern um die Frage, wie die mobilen Technologien im Spannungsfeld von Demokratisierung, Digitalisierung und Dezentralisierung effizient genutzt werden können, um in flachen Hierarchien (lohnabhängiges) ,agiles“ Arbeiten in standortübergreifenden Projekten zu ermöglichen. In der Organisationsentwicklung wird dabei besonders auf das individuelle Wahrnehmen der Arbeitsrolle bezüglich der eigenen Kompetenzen, Bedeutsamkeit/Sinnhaftigkeit, Selbstbestimmung und dem Einfluss eingegangen. Diese Individualisierung der beruflichen Rollen führt $\mathrm{zu}$ aufwendigeren Abstimmungsprozessen, da die Positionen, Aufgabenbereiche und Verantwortlichkeiten immer wieder neu ausgehandelt werden müssen. Die höheren Anforderungen an die Selbstorganisation der Mitarbeiter/innen und die Konflikte mit einer traditionell hierarchischen Unternehmenskultur bei der Umsetzung von New-Work-Initiativen erzeugen einen begleitenden Coachingbedarf zur Organisations- und Personalentwicklung, der auf den selbstorganisierten Umgang mit einer erhöhten Komplexität durch zwischenmenschliches Vertrauen eingeht (Schermuly 2019).

In einer zunehmend entgrenzten Projektarbeit wird das wechselseitige Vertrauen zu einem wichtigen Koordinationsinstrument. Hierbei wird den Menschen eine höhere Vertrauenswürdigkeit zugestanden, die den Eindruck einer ausgeprägten Fähigkeit zum Selbstmanagement vermitteln. Dazu ist auch ein persönlicher Austausch vor Ort im Unternehmen wichtig, so dass sich die mobilen Arbeitsstrukturen um einen festen, sicherheitsspendenden Organisationskern stabilisieren können. Dies erfordert das Bewusstsein für und die Fähigkeit zur Selbstbeobachtung. Solche Dispositionen sind individuell ungleich verteilt, so dass nicht jede/r Beschäftigte in gleichen Ausmaß von den freieren und dafür schlechter strukturierten Arbeitsmodellen profitieren kann, die ein höheres Maß an Selbstorganisation und -kontrolle erfordern. Bei Überforderung im entgrenzten Berufs- und Familienleben kann es daher schnell zu schlechten Arbeitsergebnissen bis zum Burnout kommen (Afflerbach und Gläsener 2016).

Die richtige Wahl der individuell passenden Arbeitsumgebung wird durch die biografisch bedingten Wechsel der Arbeitsstelle und die selbstorganisierte Entwicklung von modernen „Patchworkidentitäten“, die mit einer gelungenen Anpassung an neue soziale Unternehmensumfelder verbunden sind, immer wichtiger. Die Identität stellt eine Passung zwischen subjektiver Innen- und gesellschaftlicher Außenwelt her. In der individualisierten Gesellschaft mit ihren vielfältigen, ambivalenten Optionen der Lebensgestaltung ist dieser Prozess im gesamten Lebensverlauf nie abgeschlossen. Daher wird die passende Identitätsbildung zur permanenten, selbstorganisierten alltäglichen Identitätsarbeit in den unterschiedlichen sozialen Zusammenhängen, die in einen kohärenten Sinnzusammenhang gebracht werden müssen (Keupp 2012).

Die gelungene moderne Identitätsentwicklung verlangt aus berufsbiografischer Sicht einerseits höhere reflexive Bewältigungsleistungen im täglichen beruflichen Miteinander, aus denen sich freundschaftliche bis intime Beziehungen „,auf Zeit“ ergeben können. Die Wahl solcher persönlichen Kontakte - hier als „Partner/innen“ im Sinne der Verwirklichung gemeinsamer (impliziter) Bindungsziele während und nach der Arbeitszeit definiert - ist häufig allerdings mit verunsichernden Zuständen verbunden, welche im Spannungsfeld beruflicher und privater Interessen bewältigt werden müssen. Andererseits werden für erfolgreiche individualisierte Karriereverläufe auch verstärkt reflexive Fähigkeiten bei der Wahl des zu den eigenen fachlichen wie sozialen Präferenzen passenden Arbeitgebers vorausgesetzt. Ein attraktiver institutioneller ,(Vertrags-)Partner“ sollte neben allen materiellen Anreizen ein zum eigenen Identitätsentwurf passendes organisationskulturelles Umfeld anbieten.

Beide Seiten der „Partnerwahl“ sind mit individuellen Entwicklungsaufgaben verbunden, die eine integrierende Identitätsarbeit erfordern. In der sozial komplexen ,,agilen“ Zusammenarbeit wird Vertrauen ,unter Partnern“ oft gerade dort vorausgesetzt, wo es sich unter widersprüchlichen Arbeitsanforderungen und konfliktreichen Beziehungdynamiken kaum entwickeln kann. Die reflexive Klärung dieser zunehmenden psychischen Unsicherheiten in modernen Berufsbiografien verlangt nach dafür angemessenen und wissenschaftlich fundierten Coachingansätzen. Die interdisziplinäre Bindungsforschung kann hier wertvolle Impulse liefern, die bislang in Coachingforschung und -praxis kaum beachtet worden sind. Sie fragt nach den individuellen Faktoren, welche die psychische Sicherheit erhöhen und 
zwischenmenschliches Vertrauen in der gesamten Biografie entstehen lässt.

\section{Psychische Sicherheit durch Bindung als biografisches Leitbild der Identitätsarbeit}

Die wissenschaftliche Analyse der menschlichen Bindung durch die Bindungstheorie basiert auf den Arbeiten des britischen Psychiaters und Psychoanalytikers John Bowlby (1907-1990). Aus seiner Kritik an der allein theoretisch fundierten psychoanalytischen Objektbeziehungstheorie entwickelte er eine empirisch fundierte psychoanalytische Strukturtheorie, die in einem eklektischen Ansatz auch evolutionistische, ethologische, informationstheoretische und kognitionspsychologische Erkenntnisse berücksichtigte. Die beständige innere Bindung lässt sich danach als dauerhaftes, situationsunabhängiges psychisches ,Überlebensmuster" durch ein intensives Kontaktbedürfnis vom Nahrungs- und Sexualtrieb abgrenzen. Das äußerlich sichtbare Bindungsverhalten wird im gesamten Lebensverlauf dagegen nur in Angst- und Stresssituationen aktiviert. Bindungsverhalten beschreibt das Suchen und Bewahren von Nähe gegenüber einem als kompetenter betrachteten Menschen, um durch diesen in kritischen Situationen vor allem Zuwendungs- und Versorgungsbedürfnisse erfüllen zu können. Das Streben nach engen emotionalen Beziehungen ist ein dem Menschen angeborenes Grundbedürfnis, das Bindung zu einem lebenslang wirksamen internalisiertem Persönlichkeitsmerkmal macht (Bowlby 2008).

Die entwicklungspsychologisch ausgerichtete Bindungstheorie fragt auf Basis der grundlegenden Arbeiten von John Bowlby und Mary Ainsworth danach, wie sich beginnend aus den frühsten Eltern-Kind-Interaktionen eine Persönlichkeit entwickeln kann, die mit einem Gefühl psychischer Sicherheit ausgestattet ist. Der Begriff der psychischen Sicherheit leitet sich aus den Arbeiten der empirischen Bindungsforschung über die individuelle Verarbeitung unterschiedlicher Bindungserfahrungen eines Kindes mit seinen Eltern bzw. erwachsenen Bindungspersonen und die entsprechenden Auswirkungen auf das spätere Vertrauen in die anderen Bindungspersonen ab. Die psychische Sicherheit umfasst das Vertrauen in die schützende Nähe zur Bindungsperson bei der Exploration der Umwelt (,sichere Basis"), das mit dem Vertrauen in ihren emotionalen Rückhalt bei psychischen Belastungen z.B. durch Stress oder Krisen (,sicherer Hafen“) gekoppelt wird. Umgangssprachlich lässt sie sich als begründetes, gesundes Selbstvertrauen bezeichnen, welches durch die Interaktionen mit anderen auch dem Aufbau von Vertrauen zu anderen Personen dient. Bindung wird als imaginäres Band zwischen zwei Personen verstanden, das in den Gefühlen verankert ist und sie über Raum und Zeit miteinander verbindet. Eine sichere Bindungsrepräsentation ist das Ergebnis einer gesunden (familiären) Entwicklung, die ein Leben lang das Vertrauen auf die Unterstützung durch andere in Krisensituationen aufrechterhält. Darüber hinaus führt sie zu einer größeren Sensibilität und Offenheit beim Umgang mit anderen, indem auch bei Belastungen und Konflikten deren Perspektive eingenommen werden kann (Grossmann und Grossmann 2012).

Die individuell variierende Qualität dieses unsichtbaren emotionalen Bandes über Raum und Zeit lässt sich bindungstheoretisch auf vier grundlegende mentale Bindungsrepräsentationen zurückführen, die sich sowohl bei Kleinkindern durch experimentelle Beobachtung als auch später bei Erwachsenen durch Interviews empirisch unterscheiden lassen. Eine a) sichere Bindungsrepräsentation ist beim Erwachsenen mit einer Offenheit für negative wie positive Gefühle in den für die eigene Entwicklung als wertvoll betrachteten Bindungsbeziehungen und der ausgeprägten Reflexionsfähigkeit des kognitiven und emotionalen Perspektivenwechsels verbunden. Eine b) unsicher-distanzierende Bindungsrepräsentation in Folge emotional nicht verfügbarer bzw. zurückweisender vergangener Bindungspersonen ist im späteren Leben durch einen unflexiblen Fokus auf die Sachumwelt verbunden, der bei Gefahr die Deaktivierung der Emotionsregulierung durch die abwehrende Unterdrückung schmerzhafter negativer Gefühle, Kränkungen sowie Ängsten auslöst. Personen mit einer c) unsicher-präokkupierten (verwickelten bzw. ambivalenten) Bindungsrepräsentation weisen dagegen einen unflexiblen Fokus auf die Personenumwelt und eine übertrieben nach Nähe suchende klammernde Emotionsregulierung gegenüber der Bindungsperson auf. Klinisch relevant ist neben den drei Hauptklassifikationen ebenfalls der d) unverarbeitete Bindungsstatus, der durch sprachlich verwirrende, ängstliche oder irrationale Schilderungen von Verlusten von Bindungspersonen oder kindlicher Traumata vergeben wird und mit einer desorganisierten Bindungsrepräsentation in der Kindheit zusammenhängt (Gloger-Tippelt 2016).

In einer Meta-Analyse mit über 200 Studien und 10.500 Interviews mit dem Adult Attachment Interview (AAI) zeigt sich insgesamt eine Verteilung von $46 \%$ sicherer Bindungsmuster, $34 \%$ unsicher-distanzierte Bindungsmuster und $20 \%$ präokkupierte Bindungsmuster. Werden zudem auch unverarbeitete Bindungsmuster berücksichtigt, verschieben sich die Häufigkeiten der Bindungsmuster zu $40 \%$ sicher, $25 \%$ distanziert, $10 \%$ präokkupiert und $25 \%$ unverarbeitet oder nicht klassifizierbar. Über die unterschiedlichen Länder und Kulturen (USA, Europa, Japan, Israel) hinweg finden sich bis auf einen etwas höheren Wert unsicherer Bindungsmuster in Europa in der vierfachen Klassifikation keine bedeutenden Unterschiede dieser Verteilung. Diese weltweite Invarianz in individualistischen westlichen wie kollektivistischen östlichen Ländern deutet darauf hin, dass 
Bindung trotz der unterschiedlichen Erhebungssprachen in den landesspezifischen Interviews insgesamt ein universelles Phänomen darstellt (Bakermanns-Kranenburg und van Ijzendoorn 2009).

In der Bevölkerung überwiegen damit weltweit die unsicheren Bindungsmuster gegenüber den sicheren Bindungsrepräsentationen. Die Myriaden an inneren wie interpersonalen Konflikten, die sich aus dieser Verteilung im modernen Berufsalltag eröffnen, der auf „New Work“ setzt, stellen eine ebenso große Herausforderung wie Chance für das Coaching dar. Die interdisziplinär erforschte psychische Sicherheit kann hierbei zur explizit thematisierten Leitgröße einer durch den Coach fundiert unterstützten Identitätsarbeit der Coachees werden. In einer biografischen Perspektive eröffnet sich im Dialog der Blick auf die bisherigen und nicht mehr angemessenen bindungsbezogenen Bewältigungsmuster aktueller und zukünftiger beruflicher Herausforderungen. Ihre erfolgreiche Veränderung im Coaching hilft, das reflektierte Handeln ganzheitlich auf subjektiv ,sichere" Lösungen im Berufs- und Privatleben auszurichten.

\section{Mentalisierung als bindungstheoretisch fundierte Ressource im Coaching}

Die frühe Beziehungsumwelt an Bindungspersonen stattet das Individuum mit einem mentalen Verarbeitungssystem aus, welches mentale Beziehungsrepräsentationen hervorbringt. Bereits kleine Kinder sind dadurch in der Lage, das eigene Verhalten und das Verhalten anderer in Bezug auf mutmaßliche mentale Zustände zu deuten (Fonagy 2018). Dieses sogenannte „Mentalisieren“ beschreibt als soziale Kognition im entwicklungspsychologischen Kontext die fundamentale menschliche Fähigkeit, eigene und fremde mentale Zustände wie Gedanken und Gefühle zu beobachten, $\mathrm{zu}$ beachten und $\mathrm{zu}$ antizipieren, um eigenes Verhalten wie das anderer Menschen unter der Berücksichtigung der inneren Zustände verstehen zu können. Das Mentalisieren bildet die notwendige Grundlage für ein stabiles Selbstgefühl und konstruktive soziale Interaktionen in Beziehungen, die durch Wechselseitigkeit, Gemeinsamkeit und einem Gefühl der psychischen Sicherheit gekennzeichnet sind. Gleichzeitig wird mit Hilfe des (therapeutischen) Mentalisierens über die prägenden Bindungsbeziehungen in der eigenen Biografie selbstverstärkend auch ein besseres Mentalisieren ermöglicht (Bateman und Fonagy 2015).

Die bei stabilen Bindungsbeziehugen sich selbst verstärkende Fähigkeit zur Mentalisierung hängt somit von der psychischen Sicherheit ab. Bei Angst oder Stress ist sie allerdings bei allen Menschen ab einer bestimmten Intensität vollständig unterbrochen. Das bewusste Nachdenken über das Denken und entsprechende Erkennen von negativen Gedanken als bloße gedankliche Konstrukte, deren Realitäts- gehalt reflexiv überprüft werden kann, ist dann nicht mehr möglich. Daher bedarf es in diesen aufwühlenden Momenten einer vertrauten Bindungsperson als verständnisvolle und beruhigende sichere Basis, um die grundsätzlich bestehende eigene mentale Explorationsfähigkeit wieder zu erlangen (Holmes 2012). Andernfalls kann es bei hohem beruflichen Stress in Abhängigkeit von einem unsicheren oder sicheren Bindungsmuster jeweils früher oder später eine wiederum länger oder kürzer dauernden - unangemessene Kettenreaktion eines unbewussten „Autopiloten im Panikmodus“ geben. Solche unreflektierten selbst- bzw. karriereschädigenden Entscheidungen können gerade dann nicht mehr mentalisiert werden, wenn dies zur bewussten Gestaltung der angemessenen Reaktionen und Lösungen dringend erforderlich wäre.

In einer für uns irritierenden sozialen Situation, in der wir die mentalen Prozesse oder die Motivation von uns selbst oder anderen nicht richtig verstehen können, kommt es ohne jede Hilfe von außen leicht zu unangemessenem Verhalten. Beim komplexen Prozess des reflexiven Verstehens von unklaren mentalen Zuständen werden zunächst innere und äußere Hinweise genutzt, um sich Klarheit darüber zu verschaffen. Dabei kommt es situativ durch Missverständnisse, emotionale Erregung oder Müdigkeit leicht zu Fehlern, die zum Unbehagen im Umgang mit anderen beitragen (Bateman und Fonagy 2013). Wird diese beeinträchtigte Selbstwahrnehmung frühzeitig wahrgenommen, kann ein mentalisierungsgestütztes Coaching zu mehr oder weniger präventiven Zwecken genützt werden.

Bei sich verschärfenden Konflikten und Krisen kann ein Coach auch den dann dringend benötigten reflexiven $\mathrm{Ge}$ genpart im Rahmen einer „Rementalisierung“ übernehmen. Im Gegensatz zu den im modernen Berufsbiografien häufig räumlich weit verteilten Freundes- und Familienkreis, der zunächst akut über (digitale) Kommunikationsmedien als ,sicherer Hafen“ genutzt wird, kann er als vor Ort verfügbarer vertrauensvoller und kompetenter Gesprächpartner fungieren. Als Dialogpartner/in wirft er/sie einen fundierten Blick von außen auf die Biografie der Klienten/innen. Ein bindungstheoretisch arbeitender Coach, strebt im Verlaufe der Beziehung bewusst an, für den Coachee zu einer ,sicheren Basis“ zu werden, die sie oder ihn unterstützt, sich in seiner prinzipiell gesunden Selbst- und Fremdwahrnehmung zu stabilisieren. Das explorative Denken und Handeln kann in einem psychisch sicheren Zustand zunächst in einem geschützten Raum gemeinsam erprobt und dann wieder ganz in die eigenen Hände genommen werden.

Eine im Gespräch relativ schnell wiederkehrende bzw. von Beginn an wahrnehmbare Haltung der hohen Toleranz für Mehrdeutigkeit stellt ein für den Coach zu Prozessbeginn wichtiges Zeichen für eine solche für das Coaching im Gegensatz zur Therapie benötigten psychischen Gesundheit dar. Dauerhaft fehlendes effektives Mentalisieren verhin- 
dert dagegen, andere Personen überhaupt (emotional) $\mathrm{zu}$ verstehen oder auch nur zu beachten und daraus hilfreiche Lösungen bei Problemen abzuleiten. Die im weiteren Beratungsprozess offenbar werdenden Entdeckungen der mentalen Zustände, die den eigenen wie fremden Verhaltensweisen zu Grunde liegen und im gesamten Gesprächsverlauf gemeinsame Dialoge und neue Lösungen anregen, erfordern einen achtsamen Umgang mit der benutzten Sprache, um das forschende Bewusstsein in Bezug auf das NichtWissen der zu erkundenden subjektiven mentalen Zustände aufrechtzuerhalten. Das Üben von Achtsamkeit stärkt die Mentalisierungsfähigkeiten durch den Fokus auf den Prozess der geistigen Aktivitäten und nicht auf deren ständig wechselnden Inhalte. Durch diese innere Distanz zu den Inhalten wird die Beziehung zu den bewusst gewahr werdenden negativen Gedanken und Gefühlen verändert, die in einer offenen Grundhaltung zugelassen werden können, ohne sie zu verändern oder für eine (verfrühte) Lösungsfindung nutzen zu müssen (Allen et al. 2011). Dadurch kann die emotionale Grundqualität der geistigen Zustände wie Trauer, Ärger oder Einsamkeit als über die aktuelle Episode hinausgehendes biografisches Thema bewusst zugelassen und konstruktiv über die tieferen Ursachen mentalisiert werden, um Zugang zu neuen Perspektiven zu bekommen.

Coach und Coachee müssen sich im achtsamen Dialog immer wieder darüber klarwerden, dass sie die aktuellen mentalen Zustände des jeweils anderen ebenfalls nicht kennen und dieses Nicht-Wissen sich in einer achtsam gestalteten Coachingbeziehung auch positiv auswirken kann. Die komplexe Aufgabe, die Subjektivität der mentalen Deutung präsent zu halten und im Fluss der Erfahrungen einen gemeinsamen Zugriff zum biografischen Wissen des Coachee zu bekommen, der die Identitätsarbeit anregt, kann durch Achtsamkeit wirksam unterstützt werden.

\section{Identitätsarbeit durch Achtsamkeit im mentalisierungsgestützten Coaching}

Bezüglich der Anlässe lässt sich das Life-Coaching als psychologische Lebensberatung primär privater Themen wie Familie und Partnerschaft vom arbeitsbezogenen BusinessCoaching als Instrument der Personalentwicklung in Organisationen insbesondere für Führungskräfte unterscheiden. Letzteres wird als Coaching im engeren Sinne verstanden. Neben dem Beziehungsmanagement gegenüber den Mitarbeitern, dem Umgang mit Konflikten bei Veränderungsprozessen und der Karriereplanung kann die Variante eines Developmental-Coaching auch der individuellen Persönlichkeits- und Potenzialentwicklung dienen, um die bewusste Selbstreflexion im Sinne eines ganzheitlichen Selbstmanagements $\mathrm{zu}$ fördern. Bei beruflichen Krisensituationen (z.B. Entlassungen, Mobbing, Führungskrisen) können mit dem Coach auch Ressourcen erarbeitet und neue Perspektiven entwickelt werden. Im Rahmen eines kognitiv-behavioralen Beratungsansatzes wird im Coaching auch Achtsamkeit etwa als kognitiv-emotionale Segmentationsstrategie zur besseren Trennung von Arbeit und Privatleben nach Feierabend eingesetzt. Die aus der Psychotherapie entnommenen bewährten Konzepte sollen die Klienten/innen bei der Zielerreichung bezüglich einer besseren Work-Life-Balance wirksam unterstützten und in Form von angeleiteten dreiminütigen Atemübungen die gedankliche Fixierung auf die Arbeit in der Freizeit entspannen. Im Gegensatz zur Psychotherapie liegen gegenwärtig für das Coaching nur wenige empirische Wirksamkeitsstudien solcher Interventionen wie Anforderungen an die Coaches vor. Neben dem Ausbildungs- und Erfahrungshintergrund, Qualifizierungen und Persönlichkeitseigenschaften wie Integrität stellen die reflexiven und kommunikativen Kompetenzen des Coaches zur Vertrauensbildung über ein empathisches Verständnis bereits erfasste Erfolgsfaktoren von lösungsorientierten Coachingprozessen dar (Michel und Bickerich 2016).

In den letzten 20 Jahren wurden im Bereich der Psychotherapie zahlreiche auch für das Coaching relevante Wirksamkeitsstudien durchgeführt. Insgesamt gibt es danach keine grundsätzliche Überlegenheit einer bestimmten Therapierichtung. Eine rein kognitive Umstrukturierung der mentalen Schemata bleibt auf Dauer wirkungslos, und auch der psychoanalytische Zugang zum Unbewussten legt zwar den Fokus auf die frühkindlichen Bindungsbeziehungen, vernachlässigt jedoch, dass es keinen direkten Zugang zum Unbewussten gibt. Nur die tief ins Langzeitgedächtnis abgesunkenen Inhalte ab dem dritten Lebensjahr (,tiefes Vorbewusste") können mit Hilfe eines Coaches oder Psychotherapeuten insbesondere über die Deutung nichtverbaler Verhaltens- und Körpersignale ins Bewusstsein geholt werden können. Die kognitiven Verhaltenstherapien werden daher häufig mit emotions- und bindungsbezogenen Interventionen ergänzt, da die kognitiven Hirnzentren im oberen Stirnhirn von den limbischen Zentren getrennt sind, welche die Befindlichkeit, Emotionen, das Verhalten und unser Körpergefühl steuern. Aus neurowissenschaftlicher Sicht kommt dem allgemeinen Wirkfaktor der ,therapeutischen Allianz" eine zentrale Bedeutung zu. Sie beschreibt das Vertrauen des Patienten in den Therapeuten, dessen Überzeugung, dem Patienten helfen zu können und das Vertrauen beider in die verwendete Methode. Greift dieses Vertrauensverhältnis, wird auf beiden Seiten das „Bindungshormon" Oxytocin ausgeschüttet und führt zu einer entspannten Arbeitsatmosphäre. Das Bedürfnis leidender Menschen nach Veränderungen im Fühlen, Denken und Handeln hat das Coaching mit der Psychotherapie gemeinsam. Allerdings trennt die Schwere des Defizites und das Ausmaß der nachhaltigen Beeinträchtigung des beruflichen und privaten Lebens die Auswahl zwischen den 
beiden Interventionsverfahren. Wenn etwa die rein kontextgebundenen berufsbezogenen Störungen des Selbstbildes von Führungskräften nicht auf frühere schwere Leidenserfahrungen und entsprechende Persönlichkeitsstörungen zurückgehen oder nicht schon lange andauern, ist ein Coaching ausreichend. Dafür sollte eine spezifische Passung der ausgewählten Ansätze mit der Persönlichkeit des Coaches und den Problemen der Klienten/innen gefunden werden (Roth 2018).

Ein bindungstheoretisch fundiertes Coaching mit einem besonderen Fokus auf die mentalisierende Schilderung der aktuellen beruflichen Situation richtet sich im Gegensatz zur Therapie nicht auf grundsätzliche inneren Mentalisierungsschwächen, sondern zielt stärker auf die äußeren Stressoren organisationaler Belastungssituationen, die nur zeitweise Einbrüche normaler Reflexionsfähigkeiten verursachen. Das lösungsorientierte Vorgehen im Coaching will die situative Mentalisierungsfähigkeit auf dem grundsätzlich bestehenden reifen Niveau wiederherstellen, um den Klienten/innen ein reflektiertes Verständnis des Zusammenwirkens von situativen und persönlichen Mentalisierungseinbrüchen in ihrem beruflichen bindungsbezogenen Sozialsystem zu vermitteln. Allerdings ist derzeit noch kein praktischer Ansatz vorhanden, das theoretische Konzept des Mentalisierens explizit im Coaching zu implementieren, um die Klienten/innen anzuregen, eigene Gefühle und Gedanken wahrzunehmen und zu explorieren. Dies weist auch auf eine Lücke bei der Erforschung des Zusammenhangs von Arbeit und Bindung hin (Taubner und Kotte 2018). Diese Lücke wird seit etwa 10 Jahren verstärkt geschlossen, so dass trotz der vielen offenen Fragen zumindest erstes fundiertes Wissen für ein mentalisierungsgestütztes Coaching bereitsteht.

Der Zusammenhang zwischen Bindungsmustern, sozialem Arbeitsverhalten und Arbeitsergebnissen wird seit den 1990-Jahren erstmals untersucht. Die internationalen dyadischen Forschungsschwerpunkte analysieren die Beziehung zwischen Führungskraft und Geführten, die in ihrer karrierefördernden und unterstützenden Mentorfunktion eine groBe Ähnlichkeit zur sicheren Basis und zum sicheren Hafen besitzt. Daneben wird die Gruppenbindung in Teams erforscht, die weiterhin ein individuelles Konstrukt darstellt, auch wenn das Team als eine Art Bindungsfigur fungieren kann. Diese hat einen prägenden Einfluss auf die Mitglieder, da ängstliche Bindungsmuster zur Konfliktvermeidung und einer negativeren Selbsteinschätzung als Gruppenmitglied sowie einer schnelleren Warnung bei Gefahren führen, während distanzierte Bindungsmuster zu einer geringeren Identifikation mit dem Team und einer verringerten Bereitschaft der Berücksichtigung der Gruppenbedürfnisse beitragen. Das affektive Commitment gegenüber einer Organisation ist positiv mit einer sicheren Bindung an diese „sichere Basis“" verbunden, während eine unsichere Bin- dung wiederum ein geringes Commitment und eine höhere Rate des Verstoßes gegenüber dem psychologischen Vertrag mit dem Arbeitgeber mit sich bringt. Daher kann die Organisationsforschung von der Bindungsforschung lernen, durch welche Interventionen sich psychisch sichere Arbeitsumgebungen gestalten lassen, die mit einer erhöhten bzw. stabilisierten Bindungssicherheit einhergehen. Da das mittlerweile nicht mehr als generelles, sondern als beziehungsspezifisch wirksam betrachtete Bindungsverhaltenssystem in Stresssituationen zu verschiedenen Kollegen/innen unterschiedlich aktiviert wird und im Arbeitsleben vielfache soziale Stressoren lauern, lässt sich daraus ein in der Organisationsforschung bislang vernachlässigter organisationskultureller Zusammenhang zur Entstehung von Burnout ableiten. Vermeidend gebundene Menschen suchen sich weniger soziale Unterstützung und haben daher auch ein höheres Risiko für Burnout, wenn sie zu hohe Autonomiegrade in ihrer Arbeit haben. Eine ängstliche Bindung kann ebenfalls durch eine höhere soziale Abhängigkeit der Berufsrollen aufgefangen werden, um das erhöhte Sicherheitsempfinden zu befriedigen. Einzelne Interventionen wie Achtsamkeitsmeditationen können hierbei einen positiven Einfluss auf das psychische Wohlbefinden haben (Yip et al. 2018).

Die Achtsamkeitsmeditation kann somit den allgemeinen Wirkfaktor „therapeutische Allianz“ insbesondere für mentalisierungsgestützte Coachingsprozesse nachhaltig fördern, um die berufliche Identitätsarbeit der Klienten/ innen über die biografische Reflexion ihrer prägenden Bindungsbeziehungen erfolgreich zu unterstützten. Die reflexive Berücksichtigung von relevanten Bindungsbeziehungen in Familie, Freundeskreisen, Partnerschaften, Schule, Universität und/oder Ausbildung und vor allem im aktuell thematisierten Berufsleben verbindet als „,bindungsbiographische Identitätsarbeit“ den biographischen Kontext ihrer Entstehung mit den prägenden Wirkungen auf das gegenwärtige Denken, Fühlen und Handeln. Erst die reflektierte Kenntnis der identitätsstiftenden Bindungsbiografie erlaubt eine fundierte Identitätsarbeit zur ressourcen- wie lösungsorientierten Weiterentwicklung der eigenen Berufslaufbahn. Der Coachingfokus auf das komplexe Beziehungsgefüge in modernen Arbeits- und Organisationsformen, das sich im Zuge der Digitalisierung immer schnelllebiger gestaltet, kann von einer verlangsamten Mentalisierung durch Achtsamkeit tiefere Erkenntnisse gewinnen und damit erheblich von dieser empirisch bewährten „spirituellen Intervention“ profitieren.

Achtsamkeit gilt wie die Mentalisierung als allgemeiner Wirkfaktor in Psychotherapien. Beide Ansätze unterscheiden die objektive Realität von den subjektiven mentalen Zuständen, die diese lediglich repräsentieren und denen in einer akzeptierenden Haltung begegnet wird. Die metakognitive Ebene beim Mentalisieren umfasst das Nachdenken über das Nachdenken, während Achtsamkeit gerade kein 
aktives Nachdenken zu Gunsten des passiven Beobachtens der Gedanken wie der umfassenderen Wahrnehmung ist und dafür ein Gewahrsein des Gewahrseins (Metaawareness) ermöglicht. Durch die Mentalisierung kann die gegenwärtige Erfahrungsebene der Achtsamkeit mit sinnstiftenden Bedeutungen interpretiert werden, die in einem therapeutischen Kontext wie der Hakomi-Methode auch zukünftige und vergangene Zustände berücksichtigen lassen. Die sich mit zunehmender spiritueller Praxis immer weiter verfeinernde Kunst der möglichst ständig aufrechterhaltenen achtsamen Präsenz auf die beobachtende Wahrnehmung insbesondere auch nonverbaler unbewusster Signale des/der Klienten/in (Tracking) führt dann zu einem verbalen Ausdruck des Wahrgenommenen, der sich in einfachen und kurzen Worten auf die Gegenwart nur eines möglichen Erlebnisinhaltes bezieht und mit einer zur Exploration einladenden Frage endet (Contacting). Dadurch lassen sich auch im Coaching fürsorgliche Beziehungserfahrungen verinnerlichen, welche die bestehenden Arbeitsmodelle modifizieren. Es entsteht bei den Klienten/innen wieder eine sicherheitsspendende innere Instanz, die in Zeiten von Stress reaktiviert werden kann, um in solchen Situationen mit sich selbst mitfühlend umgehen und die Affektregulation auf einem höheren Niveau aufrechtzuerhalten (Harrer und Weiss 2016).

Im letzten Punkt geht es um die angewandte Integration dieser Erkenntnisse in einen mentalisierungs- und achtsamkeitsgestützten Coaching. Die bindungsbiografische Identitätsarbeit zur Unterstützung der selbstorganisierten Gestaltung achtsamer Begegnungen (,Mindful Dating“) verbindet die spirituelle Entfaltung der menschlichen Potenziale mit dem Denken über das Denken zur Klärung beruflicher und lebensdienlicher Anliegen.

\section{Mindful Dating als Coachingangebot einer spirituell-verbundenen Lebensführung}

Das Coaching beim sich als Work in Progress befindlichen „Mindful Dating“ (www.mindfuldating.de) entspricht der bindungstheoretischen Erkenntnis, dass wir nur in einem spezifischen psychisch sicheren Zustand auf einem für uns bislang angemessenen Niveau mentalisieren können.

Der Coachingporzess beim Mindful Dating beruht auf drei zentralen Prinzipien:

- Kopf und Herz als symbolisierte Repräsentationen verschiedener Bewusstseinszustände achtsam in die bindungsbiografisch gestützte Identitätsarbeit einbeziehen. Neben den hier vorgestellten Evidenzen aus der Bindungs- und Achtsamkeitsforschung dient die systemische Biografiearbeit einer reflektierten Entwicklung „biographischer Kompetenzen“ zur bewussten Lebensgestaltung
(Röhrbein 2019) als integrative praktische Konkretisierung der Theorie.

- Die Erkenntnisse der beiden Wissenszentren für ein gezielt zu bearbeitendes Teilanliegen im Rahmen des dialogischen Coachingprozesses zunächst möglichst getrennt erarbeiten. Dabei können ausgewählte spirituelle Methoden ressourcenorientiert integriert werden, die in stressige Zeiten ein persönliches Entwicklungsmodell für eine gelassenere Lebensbalance (Greif 2017) finden lassen.

- Dazu werden beide Ebenen des Bewusstseins am Ende einer Sitzung bzw. eines Sitzungsabschnitts lösungsorientiert integriert, um wieder psychische Sicherheit im Beruf zu gewinnen.

Im Folgenden wird die Methode anhand eines typischen autobiografischen Beispiels demonstriert, dass zum Schutze der Vertraulichkeit der Klienten/innen der Literatur entnommen wurde. Die ärztliche Profession ist zwar noch tief in hierarchischen Traditionen verankert, besitzt durch die Ökonomisierung des Gesundheitswesens und den gleichzeitig bestehenden intensiven Beziehungen zu den Patienten/ innen dafür solche stark konfligierenden Arbeitselemente, die sie als sehr anschauliches Beispiel der komplexen Identitätsarbeit in modernen Berufsbiografien erscheinen lässt. Das häufig fehlende Vertrauen durch eine sozialisierte ärztliche „Einzel-Kämpfer-Mentalität“ führt bei hoch belastenden beruflichen Krisen durch eigenes Fehlverhalten zu mangelnder Unterstützung, die nicht nur die eigene fachliche Identität bedroht, sondern auch die Versorgungsqualität beeinträchtig. Hierin liegt ein großes Potenzial für ein bindungsbiografisches Coaching im Gesundheitssystem.

\subsection{Case „Übereifer des jungen Assistenzarztes führt zu tödlichem Fehler"}

Der seit über 30 Jahren an verschiedenen Kliniken als Internist, leitender Rettungs- und Intensivmediziner und mittlerweile als Geschäftsführer des Berliner Vivantes Hospiz tätige Arzt Michael de Ridder geht in seinem autobiografischen Buch auch auf einen tödlichen Fehler in jungen Jahren als Assistenzarzt auf einer Intensivstation ein. Als eine bereits häufiger wegen ihrer Asthmaanfälle in der Klinik behandelte ältere Frau wieder auf die Intensivstation kam, weil die üblichen Therapien der Injektion von Theophyllin, Cortison und Betamimetika in Verbindung mit einer Sauerstoffsonde nicht anschlugen, wollte der seit einem halben Jahr auf Station tätige Arzt nicht untätig bleiben. In einer Mischung aus emotionaler Besorgnis über den Zustand der vertrauten Patientin, den er immer weniger ertragen konnte und dem persönlichen Ehrgeiz, eine im Vergleich zu den Kollegen wirkungsvollere Behandlung als Retter in der Not zu finden, ohne den erfahrenen Bereitschaftsarzt zu rufen, traf er die fatale Fehlentscheidung, eine künstliche Beatmung 
einzuleiten. Da er die drohenden Risiken in Folge der fehlenden Erfahrung allein nicht richtig einordnen konnte, wurde durch die Einleitung des Beatmungsschlauches der bei Asthmaanfällen besonders empfindlich auf Manipulationen im Rachen- und Kehlkopfbereich reagierende Vagusnerv derart gereizt, dass bei der erschöpften Patientin ein reflektorischer Herzstillstand auftrat und eine Wiederbelebung in Folge der geringen Sauerstoffkonzentration des Blutes bei einem Asthmaanfall scheiterte. In der reflexiven selbstkritischen Rückschau nach vielen Jahren ist es für de Ridder vor allem die Mischung aus fachlicher Selbstüberschätzung und Angst vor dem drohenden Reputationsverlust beim Wecken des Chefarztes wegen einer solchen ,dummen“ Frage, die gerade bei Assistenzärzten/innen mit weniger Erfahrung dazu führt, ihre eigene Bedürftigkeit in solchen Notfällen zu verdrängen (de Ridder 2015).

Diese psychische Unsicherheit erschwerte danach auch die ,postheroische“ Verarbeitung der Schuldgefühle, für den Tod der Patientin verantwortlich gewesen zu sein:

Ich spürte förmlich, wie sie unter meinen Händen starb; ich schaute sie an und sah das Leben aus ihren Zügen weichen - und wusste, dass meine verzweifelten, sofort einsetzenden Wiederbelebungsmaßnahmen scheitern würden. Ich war deprimiert und tief verunsichert. Eine beschämende Niederlage hatte ich mir beigebracht, und wochenlang vermied ich es, risikoreiche Eingriffe allein vorzunehmen. Manchmal erscheint mir ihr Gesicht, auch heute noch, im Traum. Sie lächelt - und bis heute finde ich für ihr Lächeln keine Antwort. (de Ridder 2015, S. 86 f.)

\subsection{Methodendemonstration zur Bearbeitung eines entsprechenden Abschiednehmens}

Das Coaching zielt auf die Begleitung und Unterstützung der individuellen Selbstklärung bezüglich der bindungsbiografischen Trauerarbeit beim Verlust von (emotional) wichtigen Patienten/innen, zu denen eine besondere Beziehung aufgebaut wurde. Die rechtlichen Fragen möglicher ärztlicher Haftung werden nicht angesprochen. Dafür kann die innerprofessionelle ärztliche Aufarbeitung durch qualitätssichernde Morbiditäts- und Mortalitätskonferenzen sinnvoll extern ergänzt werden, da die Trauergefühle dort auch in Folge des ökonomischen Zeitdrucks häufig nicht ausreichend thematisiert werden können.

Die Biografiearbeit wird hierbei als roter Kommunikationsfaden eingesetzt, um die zentralen Stationen des Trauerprozesses als den primär hier $\mathrm{zu}$ behandelnden intimen Teil der Arzt-Patienten-Beziehung im achtsamen Dialog der kognitiven Ebene des „Kopfes“ und der emotionalen Ebene des „Herzens“ zu bearbeiten und auch in einen (symbolisierten) Kontakt mit dem Toten zu treten. Der Ablauf verläuft anhand dreier Ankerpunkte auf einer Zeitlinie, die den jüngsten Beziehungsverlauf abbildet. In $\mathrm{t}=1$ wird der subjektive Zeitpunkt des Sterbens der Patientin markiert. Danach wird mit $\mathrm{t}=0$ der unumkehrbare biografische ,Nullpunkt" des fatalen Fehlers geankert und es wird erstmalig ein Dialog mit der Verstorbenen angeleitet, die als Ankerpunkt im Raum repräsentiert wird, auf dem der Klient sich dort mit ihr verbindet und deren Gefühle und Gedanken mit achtsamen Pausen als Antwort frei äußert. AnschlieBend wird $\mathrm{t}=2 \mathrm{im}$ Hier und Jetzt weiter vorne auf dem Zeitstrahl geankert und sich auf das Herz fokussiert. Der Klient wird behutsam angeleitet, eine Herzensverbindung zu der visualisierten Verstorbenen aufzunehmen und ihr intuitiv alle guten Gefühle, Empfindungen, Wünsche, Bitte um Vergebung und zuletzt auch friedvolle Gedanken zu senden.

Der Coach setzt immer wieder bewusst kleine Achtsamkeitspausen im Prozess, den der Klient in der Zukunft $(t=3)$ abschließt, in der die Ressourcen einer Arbeit frei von Schuld eingesammelt werden. Hier lässt sich bei Bedarf die bindungsbiografische Identitätsarbeit in Bezug auf ärztliche Mentoren/innen mentalisierend fortsetzten. So kann etwa der erfahrene ,väterliche“ Oberarzt, der im Praktischen Jahr als „,sicherer Hafen“ während des völlig chaotischen Nachtdienstes fungierte und der angeboten hat, auch in der fachärztlichen Weitebildung mit Rat und Tat in Form von weiterführenden Kontakten zu helfen, heute wieder angerufen werden. Zudem kann auch die spirituelle Frage thematisiert werden, inwiefern der Tod keine „Niederlage“ ist.

Die gesamte Übung schließt mit einer Achtsamkeitsmeditation ab. Sie lässt sich in modifizierter und erweiterter Form auch bei der unfreiwilligen Beendigung von wichtigen Bindungsbeziehungen zu Kollegen/innen und Vorgesetzten bei beruflichen Wechseln sowie im Privatleben durchführen. Immer dann, wenn beendete oder vergeblich erhoffte Bindungen als untrennbare Kehrseite unseres evolutionären Erbes zu Trauer, Ärger bzw. Wut oder Einsamkeitsgefühlen führen, die das eigene Denken, Fühlen und Handeln derart einschränken, dass sie in wichtigen Situationen zu einer ,bindungsbiografischen Erblast“ des temporär eingeschränkten Mentalisierens werden, kann ein solches Coaching weiterhelfen, neue ganzheitliche Lösungen zu finden.

Interessenkonflikt M. Krohn gibt an, dass kein Interessenkonflikt besteht.

Open Access Dieser Artikel wird unter der Creative Commons Namensnennung 4.0 International Lizenz veröffentlicht, welche die Nutzung, Vervielfältigung, Bearbeitung, Verbreitung und Wiedergabe in jeglichem Medium und Format erlaubt, sofern Sie den/die ursprünglichen Autor(en) und die Quelle ordnungsgemäß nennen, einen Link zur Creative Commons Lizenz beifügen und angeben, ob Änderungen vorgenommen wurden. 
Die in diesem Artikel enthaltenen Bilder und sonstiges Drittmaterial unterliegen ebenfalls der genannten Creative Commons Lizenz, sofern sich aus der Abbildungslegende nichts anderes ergibt. Sofern das betreffende Material nicht unter der genannten Creative Commons Lizenz steht und die betreffende Handlung nicht nach gesetzlichen Vorschriften erlaubt ist, ist für die oben aufgeführten Weiterverwendungen des Materials die Einwilligung des jeweiligen Rechteinhabers einzuholen.

Weitere Details zur Lizenz entnehmen Sie bitte der Lizenzinformation auf http://creativecommons.org/licenses/by/4.0/deed.de.

\section{Literatur}

Afflerbach, T., \& Gläsener, K. M. (2016). New Ways of Working - Vertrauen und Selbstmanagement in einer digitalisierten Arbeitswelt. In B. Badura, et al. (Hrsg.), Fehlzeiten-Report 2016 (S. 171-181). Berlin, Heidelberg: Springer.

Allen, J.G., et al. (2011). Mentalisieren in der psychotherapeutischen Praxis. Stuttgart: Klett-Cotta.

Bakermans-Kranenburg, M.J., \& van Ijzendoorn, M.H. (2009). The first 10.000 adult attachment interviews: distributions of adult attachment representations in clinical and non-clinical groups. Attachment \& Human Development, 11(3), 223-263.

Bateman, A. W., \& Fonagy, P. (2015). Vorwort. In A. W. Bateman \& P. Fonagy (Hrsg.), Handbuch Mentalisieren (S. 13-20). Gießen: Psychosozial-Verlag.

Bateman, A., \& Fonagy, P. (2013). Mentalisierungsbasierte Therapie und Borderline-Persönlichkeitsstörung. In J.F. Clarkin, et al. (Hrsg.), Psychodynamische Psychotherapie der Persönlichkeitsstörungen - Handbuch für die Klinische Praxis (S. 184-205). Stuttgart: Schattauer.

Bowlby, J. (2008). Bindung als sichere Basis. München: Ernst Reinhardt.

Fonagy, P. (2018). Bindungstheorie und Psychoanalyse (4. Aufl.). Stuttgart: Klett-Cotta.

Gloger-Tippelt, G. (2016). Das Adult Attachment Interview: Durchführung und Auswertung. In G. Gloger-Tippelt (Hrsg.), Bindungen im Erwachsenenalter (3. Aufl. S. 93-112). Bern: Hogrefe.
Greif, S. (2017). Stress und Stressmanagement im Coaching. In S. Greif, et al. (Hrsg.), Handbuch Schlüsselkonzepte im Coaching (S. 1-10). Berlin, Heidelberg: Springer.

Grossmann, K., \& Grossmann, K.E. (2012). Bindungen - das Gefüge psychischer Sicherheit (5. Aufl.). Stuttgart: Klett-Cotta.

Harrer, M. E., \& Weiss, H. (2016). Wirkfaktoren der Achtsamkeit. Stuttgart: Schattauer.

Holmes, J. (2012). Sichere Bindung und Psychodynamische Therapie. Stuttgart: Klett-Cotta.

Keupp, H. (2012). Identität und Individualisierung: Riskante Chancen zwischen Selbstsorge und Zonen der Verwundbarkeit - sozialpsychologische Perspektiven. In H.G. Petzold (Hrsg.), Identität (1. Aufl. S. 77-106). Wiesbaden: VS.

Michel, A., \& Bickerich, K. (2016). Berufliche Entwicklung steuern und Erfolg fördern: Mentoring und Coaching. In K. Sonntag (Hrsg.), Personalentwicklung in Organisationen (4. Aufl. S. 561-600). Göttingen: Hogrefe.

de Ridder, M. (2015). Welche Medizin wollen wir? München: Deutsche Verlags-Anstalt.

Röhrbein, A. (2019). Und das ist noch nicht alles. Systemische Biografiearbeit. Heidelberg: Carl-Auer.

Roth, G. (2018). Coaching und Neurowissenschaften. Organisationsberatung Supervision Coaching, 25, 109-120.

Schermuly, C. (2019). New Work und Coaching - psychologisches Empowerment als Chance für Coaches. Organisationsberatung Supervision - Coaching, 26, 173-192.

Taubner, S., \& Kotte, S. (2018). Mentalisierung im Coaching. In S. Greif, et al. (Hrsg.), Handbuch Schlüsselkompetenzen im Coaching (S. 353-362). Berlin, Heidelberg: Springer.

Wanka, A. (2020). Doing Transitions: Perspektiven und Ziele einer reflexiven Übergangsforschung. In A. Walther (Hrsg.), Reflexive Übergangsforschung (S. 11-36). Opladen, Berlin, Toronto: Barbara Budrich.

Yip, J., et al. (2018). Attachment theory at work: a review and direcrtions für future research. Journal of Organizational Behavior, 39, 185-198.

Publisher's Note Springer Nature remains neutral with regard to jurisdictional claims in published maps and institutional affiliations. 